OPEN ACCESS

Edited by:

Han Xu,

Zhejiang University, China

Reviewed by:

Laura Baroncelli,

National Research Council (CNR), Italy

Gabriele Sansevero,

Fondazione Umberto Veronesi, Italy

*Correspondence:

Hirofumi Morishita

Hirofumi.morishita@mssm.edu

†These authors have contributed equally to this work

Received: 05 March 2021 Accepted: 19 April 2021

Published: 12 May 2021

Citation

Bicks LK, Peng M, Taub A, Akbarian S and Morishita H (2021) An Adolescent Sensitive Period for Social Dominance Hierarchy Plasticity Is Regulated by Cortical Plasticity Modulators in Mice.

Front. Neural Circuits 15:676308. doi: 10.3389/fncir.2021.676308

\section{An Adolescent Sensitive Period for Social Dominance Hierarchy Plasticity Is Regulated by Cortical Plasticity Modulators in Mice}

\author{
Lucy K. Bicks ${ }^{1,2,3,4,5}$, Michelle Peng ${ }^{1,2,3,4,5+}$, Alana Taub ${ }^{1,2,3,4,5+}$, Schahram Akbarian ${ }^{1,2,5}$ and \\ Hirofumi Morishita ${ }^{1,2,3,4,5 *}$ \\ ${ }^{1}$ Department of Psychiatry, Icahn School of Medicine at Mount Sinai, New York, NY, United States, ${ }^{2}$ Department \\ of Neuroscience, Icahn School of Medicine at Mount Sinai, New York, NY, United States, ${ }^{3}$ Department of Ophthalmology, \\ Icahn School of Medicine at Mount Sinai, New York, NY, United States, ${ }^{4}$ Mindich Child Health and Development Institute, \\ Icahn School of Medicine at Mount Sinai, New York, NY, United States, ${ }^{5}$ Friedman Brain Institute, Icahn School of Medicine \\ at Mount Sinai, New York, NY, United States
}

Social dominance hierarchies are a common adaptation to group living and exist across a broad range of the animal kingdom. Social dominance is known to rely on the prefrontal cortex (PFC), a brain region that shows a protracted developmental trajectory in mice. However, it is unknown to what extent the social dominance hierarchy is plastic across postnatal development and how it is regulated. Here we identified a sensitive period for experience-dependent social dominance plasticity in adolescent male mice, which is regulated by mechanisms that affect cortical plasticity. We show that social dominance hierarchies in male mice are already formed at weaning and are highly stable into adulthood. However, one experience of forced losing significantly reduces social dominance during the adolescent period but not in adulthood, suggesting adolescence as a sensitive period for experience-dependent social dominance plasticity. Notably, robust adolescent plasticity can be prolonged into adulthood by genetic deletion of Lynx1, a molecular brake that normally limits cortical plasticity through modulation of cortical nicotinic signaling. This plasticity is associated with increased activation of established nodes of the social dominance network including dorsal medial PFC and medial dorsal thalamus evidenced by increased c-Fos. Pharmacologically mediated elevation of cortical plasticity by valproic acid rapidly destabilizes the hierarchy of adult wildtype animals. These findings provide insight into mechanisms through which increased behavioral plasticity may be achieved to improve therapeutic recovery from psychiatric disorders that are associated with social deficits.

Keywords: social hierarchy, plasticity, development, prefrontal cortex, thalamus

\section{INTRODUCTION}

Adult mice living in social groups form naturally occurring dominance hierarchies which are highly stable across time (Lindzey et al., 1961; Wang et al., 2011; Williamson et al., 2016; Zhou et al., 2018). Hierarchy formation and maintenance is a complex behavior that relies on recognition of dominance relationships of others and behavioral plasticity in response (Wang et al., 2014; 
Bicks et al., 2015; Zhou et al., 2018). Previous research has demonstrated that dominance rank is dependent on prefrontal cortex (PFC) circuitry acting in conjunction with midline thalamic structures (Wang et al., 2011; Zhou et al., 2017; Kingsbury et al., 2019; Nelson et al., 2019). However, very little is known about the mechanisms that establish and maintain the stability of these hierarchies. While dominance is a behavioral trait of an individual, the stability of the hierarchy is a property of the group as a whole and is dependent on the change in dominance status of all the individuals within the group. Here we set out to examine the neural mechanisms in control of hierarchy stability as a model for social behavior plasticity in mouse.

While much research in primary sensory cortical areas has outlined the specific mechanisms for regulating experiencedependent development and plasticity of circuitry mediating sensory processing (Hensch, 2004; Morishita and Hensch, 2008), mechanisms regulating experience-dependent development of PFC circuits are still poorly understood. Social experience in the post-weaning juvenile and adolescent periods has profound effects on the development of adult PFC circuitry and social behaviors (Makinodan et al., 2012; Bicks et al., 2020; Morishita, 2020; Yamamuro et al., 2020), suggesting that the circuitry regulating hierarchies may be sensitive to social experience during this period. Whether hierarchies are more plastic during development, or whether modulators of cortical plasticity impact hierarchy plasticity is as of yet unknown.

Several molecules have been identified as cortical plasticity modulators in primary sensory cortical areas, including several molecular "brakes" which actively limit plasticity as the critical period closes (Bavelier et al., 2010). One such plasticity brake is Lynx1, a nicotinic modulator that facilitates closure of the visual critical period (Morishita et al., 2010). Lynx1 knockout (KO) mice, have an open-ended critical period and therefore provide an opportunity to study the effects of elevated cortical plasticity in the adult (Morishita et al., 2010; Bukhari et al., 2015; Sajo et al., 2016; Takesian et al., 2018). Pharmacological interventions, such as Valproic Acid treatment, can reopen critical period cortical plasticity in adults, allowing for interrogation of the impacts of high cortical plasticity on adult behavior (Putignano et al., 2007; Silingardi et al., 2010; Yang et al., 2012; Gervain et al., 2013). For example, in humans, administration of VPA reopens a critical period for auditory learning, allowing for acquisition of absolute pitch in adults (Gervain et al., 2013). It is currently unknown whether critical periods in association cortical areas (including the PFC) are regulated by mechanisms that are shared or distinct to those that regulate primary sensory critical periods. Understanding the mechanisms that regulate plasticity of the PFC and behaviors regulated by the PFC could provide invaluable tools to leverage plasticity mechanisms to improve cognitive and social functioning in adults.

Here we examine the development of mouse social dominance hierarchies and their regulation by critical period modulators, Lynx1, and VPA. We show that baseline mouse hierarchies are stable at an early developmental stage, but show experiencedependent plasticity in response to an experience of losing during the adolescent window. Experience-dependent plasticity is dampened in the adult animal but can be extended into adulthood by genetic absence of Lynx1, while unmasking plasticity in adulthood using the pharmacological treatment VPA leads to destabilization of the baseline hierarchy in mice. Our findings show the first evidence that modulators of sensory critical period plasticity also regulate social behavior plasticity.

\section{MATERIALS AND METHODS}

\section{Animals}

Juvenile and adult (14 days to 5 months after birth) WT (C57Bl6, Charles River, Wilmington, MA, United States) and adult Lynx1KO (gifted by Dr. Nathaniel Heintz: Rockefeller University) male mice were maintained on a 12-h light/dark (LD) cycle and had access to food and water ad libitum. Mice were group housed, 4 in a cage, for at least two weeks after shipping prior to any behavioral experimentation. Information on original litter size was not available. All experiments were approved by the Icahn School of Medicine at Mount Sinai Ethical Committee for animal research.

\section{Behavior}

Mice were first habituated to the tube and the testing environment by placing the subject at one end of the tube and allowing each mouse to run the length of the tube. The direction was alternated between turns. Habituation was completed after successful completion of the task four times. Each day of testing involved pairwise assessment of all possible matches in the tube-test (six total matches). The order of the matches was determined randomly and varied each testing session. Testing began following one training session. Each match was started when both mice fully entered the tube on opposite sides, and ended when one mouse had forced the other to retreat with all four paws out of the plastic tube. Mice were tested in a Plexiglas tube measuring $1 \mathrm{ft}$ in length. The diameter was adjusted depending on the developmental age $(0.75-1.25$ inches internal diameter, Interstate Plastics). Following every trial, the apparatus was cleaned with a cleaning MB-10 solution (Virkon S, Dupont, Wilmington, DE, United States). Developmental tests were conducted weekly, while Valproic Acid (VPA) and Lynx1KO experiments were conducted every other day. To assess experience-dependent hierarchy plasticity, baseline hierarchies were established based on every other day testing for three days, and rank was determined based on number of wins on the final day. The following day, the top ranked animal was forced to lose to the third ranking animal by placing a stopper at the end of the tube, such that the top rank animal would be forced to back out. We assessed hierarchies the following day by again testing pairwise dominance between all pairs in the tube-test.

\section{Valproic Acid Administration}

Valproic Acid (VPA), (200 mg/kg, dissolved in sterile saline) or Saline was injected i.p. every $12 \mathrm{~h}$ for 8 days.

\section{Statistical Analyses}

David's scores (DSs) were assessed using the EloRating R package (Gammell et al., 2003). Generalized linear models were used 
to assess effects of treatment using the "glmer" function of the lme4 R package. Generalized linear models included the following random effects: animal nested within cage and test date. Generalized linear models including treatment (genotype or drug treatment) as an effect were compared to reduced models not including treatment. Differences between Pearson's correlations were assessed using the cocor $\mathrm{R}$ package to assess Pearson and Filon's z.

\section{Immunohistochemistry}

Mice were deeply anesthetized with isoflurane and perfused transcardially with $0.1 \mathrm{M}$ phosphate-buffer saline $(\mathrm{PBS}, \mathrm{pH}=7.4$ ) followed by $4 \%$ paraformaldehyde in PBS. Brains were removed and transferred to the same fixative. 4-6 $\mathrm{h}$ later they were cryoprotected in 30\% sucrose in PBS.

All rank one mice were analyzed from 7 cages. Coronal brain sections $(35 \mu \mathrm{m})$ were collected in serial sections using a 24 well plate using a cryostat (Leica). Samples were washed two times with TBS for $10 \mathrm{~min}$ and then blocked with (1\% BSA/0.25\% triton-X 100/TBS) for $1 \mathrm{~h}$ at RT. Sections were then exposed to primary antibody (rabbit anti-c-Fos antibody 1:5000) in.1\% triton-X $100 / \mathrm{TBS}$ at $4^{\circ} \mathrm{C}$ on a rotator for $20 \mathrm{~h}$. Sections were then washed with a blocking buffer for $10 \mathrm{~min}$, three times at RT. Next, sections were exposed to the secondary antibody (donkey anti-rabbit IgG1:400) (Life Technologies, A21206, Lot\#1182675) in. $25 \%$ triton-X/TBS at RT for $2 \mathrm{~h}$. Slices were washed in TBS for $10 \mathrm{~min}$ two more times. Sections were transferred onto glass slides, air dried, mounted with Fluoromont-G Dapi (Southern Biotech) and coverslipped.

\section{Imaging and Quantification}

Images were acquired on a LSM780 Microscope using Zen 2012 software. Tilescans of mPFC slices were captured and Images were analyzed using Fiji. Using the DAPI channel, brain regions of the mPFC were drawn. Bregma range [ -0.08 to 3.45] was included in the calculation. Dorsal Anterior Cingulate (dACC) and the Mediodorsal nucleus of the thalamus (MD) were drawn according to the Allen Brain Atlas. The cFos channel was selected: the image was converted to an 8bit type, noise was removed and background was subtracted. Each region was individually selected and the number of nuclei counts was recorded. Imaging and quantification was performed by a blinded experimenter.

\section{RESULTS}

\section{Experience-Dependent Plasticity of Social Dominance Hierarchy in Adolescent Mice}

We first set out to evaluate the baseline stability of social hierarchy in mice from the juvenile period following weaning to adulthood. We assessed dominance between all pairs of male mice within a cage of 4 mice in the tube-test (Lindzey et al., 1961) (Figure 1A). Dominance scores derived from behavior in the tube test have been previously shown to correlate well with measures of dominance produced by other assays such as scent marking in the presence of a female and competition for a warm spot (Wang et al., 2011; Wang et al., 2014). A social dominance score for each mouse was quantified by calculating DSs (David, 1987) which are based on the number of wins, and the relative numbers of wins and losses of the opponent. With 4 mice in a cage, the DS ranges from -3 to 3 , with a score of -3 representing a mouse who lost all matches and a score of three given to a mouse that wins all matches. Tube tests were repeated weekly following weaning through adulthood (Figure 1B). To assess the stability of social hierarchy across development, we first analyzed the weekly change in DSs (Figure 1C). We observed a modest, but not significant, decrease in the weekly change in DSs across development (Figure 1C). Even in the juvenile period, DSs were stable, with an average change of less than one, which is less than a single rank change in a cage of four mice. For example, in a cage with no ties, a rank 3 to rank 4 change is equivalent to a change in DS from -1 to -3 . We further examined the specific ranks (based on the average DS throughout the testing period) and saw that the rank 1 in particular was highly invariable (Figure 1D). This finding demonstrates the presence of stable hierarchies already formed during development (Figures 1C,D). Critical period plasticity is often not observed under baseline conditions, and is characterized by experience-dependent changes (Gordon and Stryker, 1996). Therefore, we tested if a forced experience of losing could disrupt the rank of adolescent mice. After determining baseline rank in each cage, we manipulated the outcome of the tube-test trial by blocking one end of the tube such that the rank 1 animal would need to back out and the lower rank animal (rank 3) would need to walk out, creating an experience of "loss" for the rank 1 and an experience of "win" for the rank 3 animal, and we compared this to an unmanipulated test ("natural outcome") (Figures 1E-G). We found that adolescent mice (p35) showed significantly reduced DSs one day following forced loss, compared with the natural loss controls (Figure 1H). Overall, these findings suggest that stable social hierarchies are formed early in the juvenile period, but that these hierarchies are plastic to experience during the adolescent period.

\section{Prolonged Experience-Dependent Plasticity of Social Dominance Hierarchy Into Adulthood by Genetic Deletion of Lynx1, A Cortical Plasticity Regulator}

We next aimed to determine the mechanism supporting adolescent experience-dependent dominance plasticity. Given that hierarchies show experience-dependent changes during adolescence (Figure 1H), we hypothesized that hierarchy stability may be regulated by modulators of cortical plasticity, which are typically high during juvenile windows and decrease in adulthood. To test this, we leveraged a model of open-ended critical period cortical plasticity, the Lynx1KO mouse. Lynx1 is a nicotinic modulator that increases during adolescence, providing a brake on cortical plasticity in adults. Adult Lynx1KO mice have an open-ended critical period for cortical plasticity, allowing for juvenile-like plasticity in primary sensory cortical areas in adult animals (Morishita et al., 2010; Bukhari et al., 2015; Sajo et al., 2016; Takesian et al., 2018). To test whether social 
A
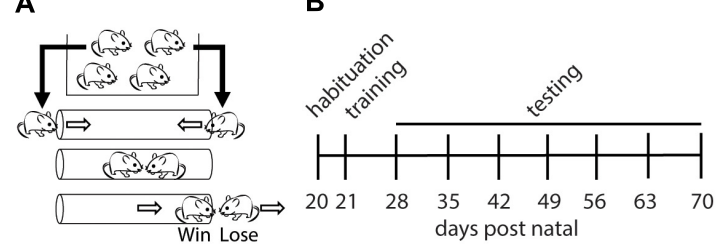

E Adolescent

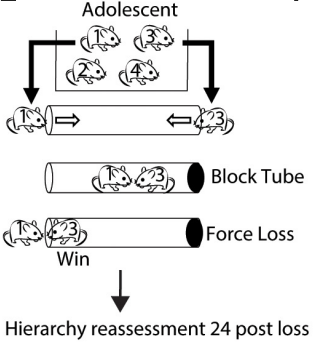

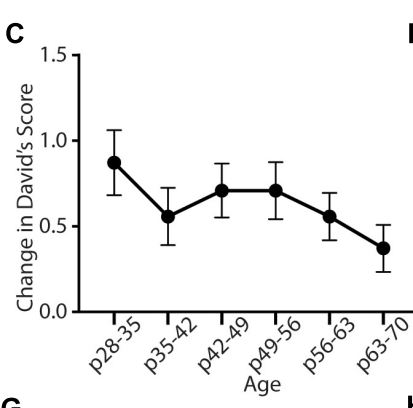

G

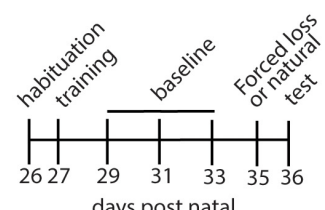

days post natal
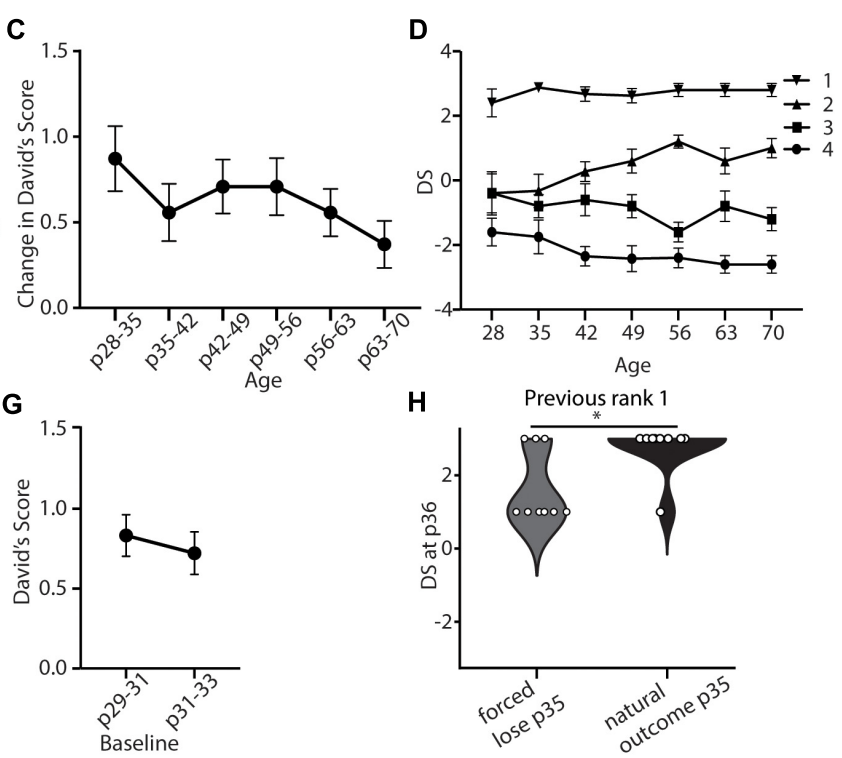

FIGURE 1 | Dominance shows developmentally regulated experience-dependent plasticity. (A) Mice within a cage were assessed in the tube test using a round-robin design to assess dominance between each pair in a cage. (B) Timeline showing habituation to the tube and testing environment and training, followed by weekly tests of hierarchy across development. (C) Weekly changes in average David's Score (DSs) across development (generalized linear model, $p=0.739, n=43$ mice in 11 cages) (D) DS for each rank assigned based on the average DSs across the testing period. (E) Experience-dependent changes in hierarchy for adolescent mice were assessed by altering the outcome of the rank 1 vs. 3 match-up by blocking the tube, forcing the rank 1 animals to lose. Hierarchies were reassessed $24 \mathrm{~h}$ later ( $n=72$ mice in 18 cages). ( $F$ ) Timeline showing habituation to the tube and testing environment, training, three baseline tests of hierarchy every other day, followed by forced loss manipulation or natural outcome and dominance status reassessment. (G) Baseline hierarchies showed no significant differences in change in David's Score between p29-31 and p31-33 (generalized linear model, $p=0.614, n=72$ mice from 18 cages). (H) Adolescent rank 1 mice that experienced forced loss showed significantly lower DSs compared with mice that experienced the natural outcome (Wilcoxon signed rank test, ${ }^{*} p=0.02, n=9$ mice from 18 cages).

hierarchies may be governed by shared neurobiology underlying critical period cortical plasticity in primary sensory areas, we first assessed baseline hierarchy stability in adult WT and Lynx1KO mice following habituation and training (Figure 2A). There were no significant differences in baseline change in DSs across groups, mirroring adolescent data (Figure 2B). Next, we employed our "forced-lose" assay to assess experiencedependent changes in dominance in Lynx1KO and WT adult male mice (Figure 2C). We then re-tested the hierarchy $24 \mathrm{~h}$ later and found that compared to adult WT rank 1 mice, adult Lynx1KO rank 1 mice showed significantly reduced DS $24 \mathrm{~h}$ after unexpected experiential loss (Figure 2F). These findings demonstrate significant plasticity in social hierarchy based on experience in adult animals with open-ended critical period for cortical plasticity, suggesting modulators of cortical plasticity may regulate dominance hierarchy stability.

\section{Brain Regions Involved in Control of Social Dominance Show Differential Response to Loss in a Model of Open-Ended Cortical Plasticity}

Next, we aimed to determine the brain regions associated with the plasticity of social hierarchy regulated by Lynx1. Convergent evidence shows regions of the dorsal medial PFC, including the dorsal Anterior Cingulate Cortex (dACC), acting in concert with the medial dorsal thalamus (MD) are essential for regulating rank within a hierarchy (Wang et al., 2011; Zhou et al., 2017; Nelson et al., 2019). We therefore tested whether these regions show differential activation after experiencing an unexpected loss in the tube test between WT animals, who go on to maintain their rank, and Lynx1KO animals, whose rank decreases in the following $24 \mathrm{~h}$ (Figure 2D). We perfused Lynx1KO and WT rank 1 animals that had experienced a forced loss 90 min prior in order to assess early immediate gene c-Fos activation in the dominance network, including the dACC and MD (Figures 2E,F). We found that Lynx1KO animals had increased c-Fos activation in both the $\mathrm{dACC}$ and the MD, suggesting activation of the dominance network may be important for encoding the experience of loss leading to a subsequent decrease in dominance (Figures 2E,F). Overall, our findings suggest that experience-dependent plasticity of social hierarchy in adult mice is limited by the cortical plasticity modulator Lynx1 and is associated with corticothalamic network activity.

\section{A Pharmacological Modulator of Cortical Plasticity, Valproic Acid, Induces Plasticity in Adult Social Hierarchy}

We next set out to test whether regulation of the stability of mouse dominance hierarchies is broadly regulated by shared neurobiology in control of cortical plasticity, or is specifically regulated by nicotinic acetylcholine modulation by Lynx1. To this end, we injected WT mice with Valproic Acid (VPA), a 
A
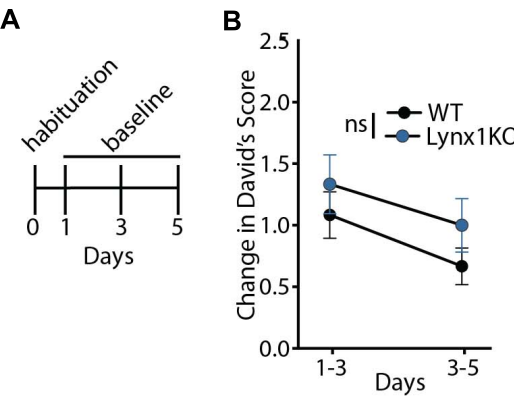

E
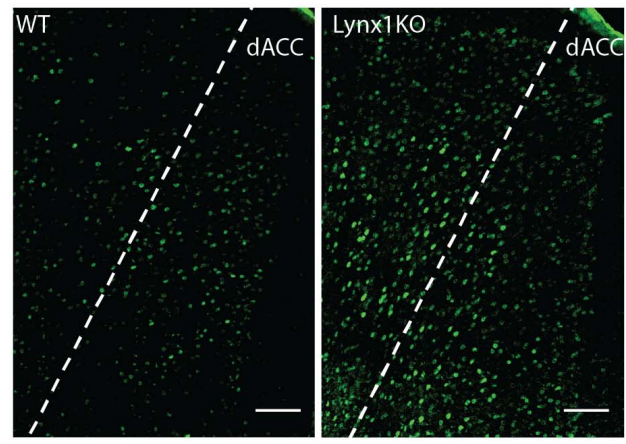

dACC

Previous rank 1

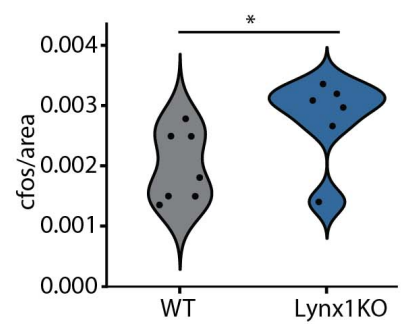

C

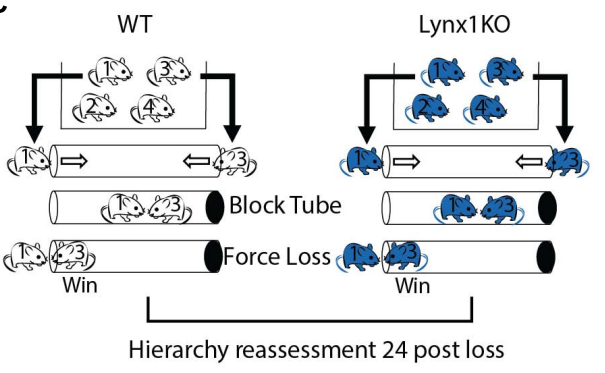

F
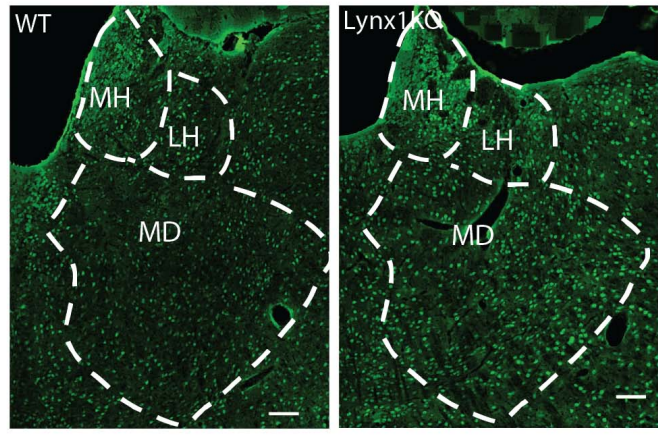

MD

Previous rank 1

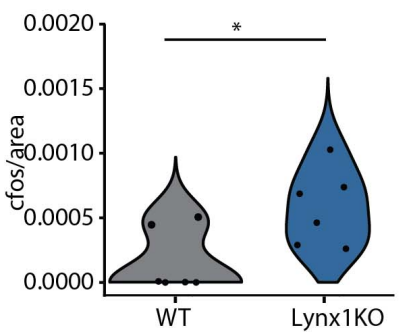

FIGURE 2 | Cortical plasticity modulator Lynx1 limits experience-dependent plasticity of social hierarchy in adult mice. (A) Adult WT or Lynx1KO mice were tested in a round-robin design to assess dominance between all pairs in a cage. Mice were habituated to the tube and then baseline hierarchy was assessed every other day for 5 days. Baseline day 1 was training. (B) Baseline hierarchies showed no significant differences in change in David's Score (DS) between genotypes (generalized linear model, $p=0.323, n=36$ Lynx1 KO from 9 cages, $n=48$ WT from 12 cages). (C) Experience-dependent changes in hierarchy were assessed by altering the outcome of the rank 1 vs. 3 match-up by blocking the tube, forcing the rank 1 animals to lose. Hierarchies were reassessed $24 \mathrm{~h}$ later. (D) WT previous rank $1 \mathrm{mice}$ maintained their rank following loss while Lynx1KO mice showed significantly reduced DS 24 h post loss (Wilcoxon signed rank test, ${ }^{*} p=0.03, n=7$ WT mice, 7 Lynx1KO mice). (E) Dorsal Anterior Cingulate (dACC) shows significantly increased c-Fos labeling in adult Lynx1 KO mice following forced loss (two-tailed un-paired student's $t$-test, ${ }^{*} p=0.05, n=7$ WT mice, $n=6$ Lynx1KO mice). (F) Mediodorsal thalamus (MD) shows significantly increased c-Fos in adult Lynx1 KO mice following forced loss (two-tailed unpaired student's $t$-test, ${ }^{*} p=0.02, n=7 \mathrm{WT}$ mice, $n=6$ Lynx1KO mice).

pharmacological manipulation that is known to re-open adult visual and auditory critical periods (Putignano et al., 2007; Silingardi et al., 2010; Yang et al., 2012; Gervain et al., 2013). Mice were injected twice daily for 8 consecutive days and underwent the tube test every other day (Figure 3A). We found that VPA treatment significantly increased the change in DS, indicating destabilization of the baseline hierarchy (Figure 3B). We then allowed all cages to re-stabilize their hierarchies with a 2 -week washout period with no handling, re-established baseline DSs, and began saline injections twice daily for eight consecutive days (Figure 3A). Saline injection did not destabilize hierarchies, showing a specific effect of VPA on hierarchy stability. DSs were highly correlated in both baseline periods (Figures 3D,E top panels), but following VPA treatment, correlations across tests began to breakdown, showing uncorrelated hierarchies after one week of VPA treatment, but not following Saline treatment (Figures 3D,E middle panel). This led to a significant difference between strength of correlation at baseline and following VPA treatment, but not following Saline treatment (Figures 3D,E bottom panel). Therefore, adult re-opening of juvenile-like cortical plasticity with VPA treatment destabilizes hierarchies without further manipulation (such as unexpected experience of losing which was necessary to induce plasticity in hierarchy in adult Lynx1KO mice and adolescent WT mice). This difference may reflect the timing and the nature of manipulations; while Lynx1KO results in open-ended cortical plasticity, VPA 
administration re-open plasticity in the adult after the critical period had closed. Alternatively, the difference in findings between Lynx1KO and VPA administration could be due to the nature of the manipulation: While Lynx1KO removes one of many brakes on cortical plasticity (Bavelier et al., 2010), VPA administration is a broad manipulation that has multiple targets. Overall, our findings support the model that the stability of mouse social dominance hierarchies is broadly regulated by the neurobiological mechanisms that control cortical plasticity.

\section{DISCUSSION}

The results from this study show that a sensitive period for experience-dependent social dominance plasticity of male mouse social dominance hierarchies is regulated by molecular modulators of cortical plasticity that are known to regulate critical periods in sensory cortex (Figure 4). Lynx1, a developmentally regulated brake on critical period plasticity, regulates experience-dependent change in social dominance rank, as hierarchies of Lynx1KO mice are destabilized by a single experience of forced-losing while WT hierarchies remain intact. Forced losing activates regions known to be involved in dominance such as the $\mathrm{dmPFC}$ and midline thalamic structures. Pharmacological re-opening of critical period cortical plasticity rapidly destabilizes hierarchy structures at baseline, showing that, in the adult animal, decreased plasticity helps to maintain a stable dominance relationship within the cage. Our findings provide evidence of cortical plasticity modulators directly altering behavior in a social context and shed light on our understanding of the developmental tradeoff between behavioral stability and plasticity in the face of changing contexts.

Previous studies on mouse social dominance hierarchies have studied ways in which rank can be changed by manipulating molecular and circuit mechanisms (Timmer and Sandi, 2010; Wang et al., 2014; Yamaguchi et al., 2017a,b; Zhou et al., 2018). For example, rank can be increased by increasing AMPA receptor mediated synaptic transmission within the dmPFC (Wang et al., 2011), or by manipulating the MD to dmPFC projection (Zhou et al., 2017; Zhou et al., 2018; Nelson et al., 2019). These studies have demonstrated that a dominant animal can lose its' status, or a subordinate animal can gain rank. In our study, instead of focusing on the dominance rank of individual animals per se, we studied the rank stability within a group hierarchy. Unlike manipulations previously discussed, adult manipulations of cortical plasticity in this study change the overall stability of the hierarchy, leading to high amounts of rank change regardless of the direction of change. This study therefore adds to a growing body of knowledge about the mechanisms mediating male mouse hierarchies by providing evidence that increased cortical plasticity leads to hierarchy destabilization. While this study manipulated cortical plasticity broadly, we observed convergence onto known circuits that mediate dominance rank, suggesting increased activation of these circuits is associated with higher plasticity of the hierarchy within the cage.

Our results show that a genetic brake on plasticity, Lynx1, can be removed leading to high experience-dependent dominance

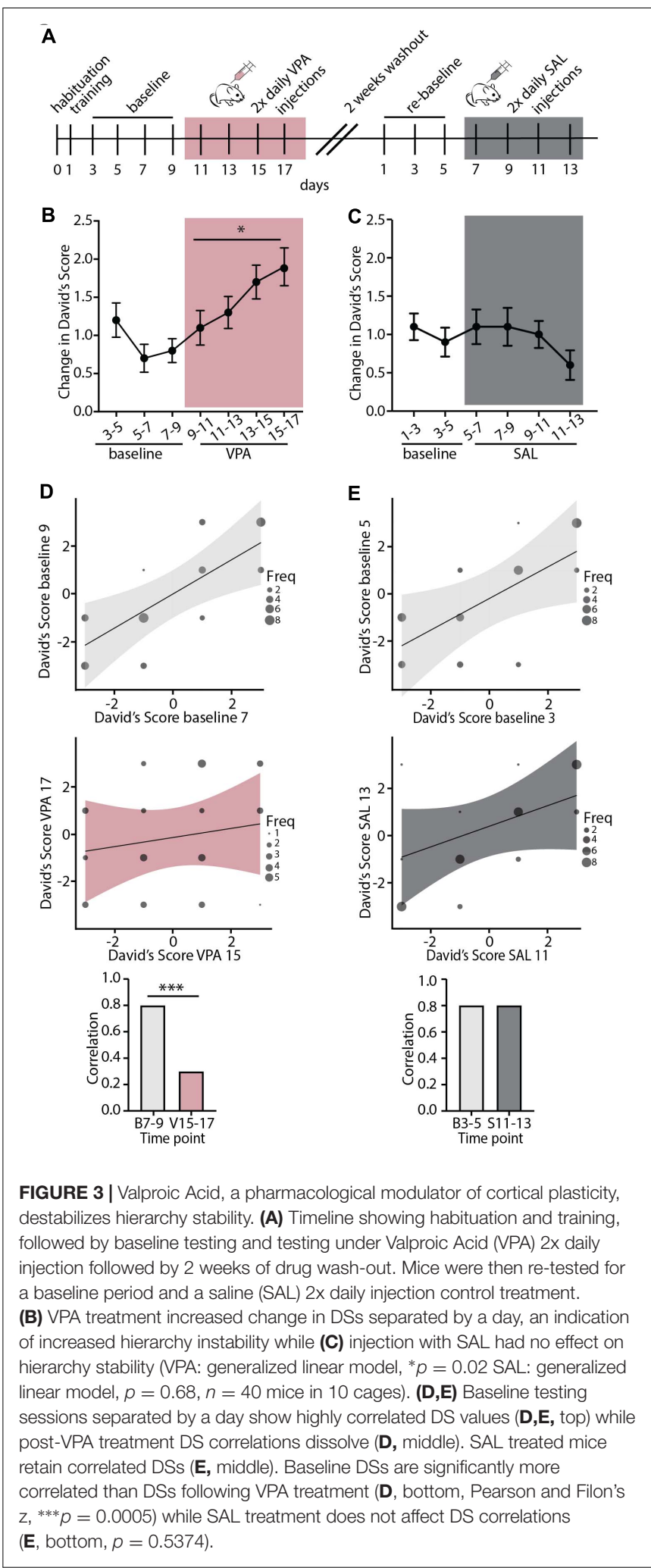

plasticity in adult male mice. As Lynx1 is a modulator of nicotinic signaling in the cortex and desensitizes cells to acetylcholine responses (Miwa et al., 1999; Ibañez-Tallon et al., 2002; 


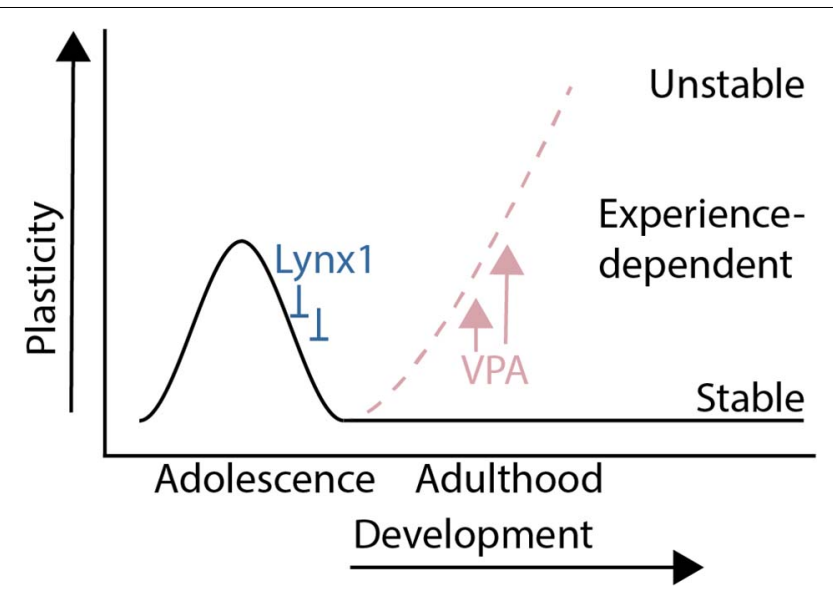

FIGURE 4 | Summary: A sensitive period for adolescent social dominance plasticity is regulated by cortical plasticity modulators.

Miwa et al., 2006), our findings suggest a key role of nicotinic signaling in regulating hierarchy plasticity. Previous studies have shown that nicotinic acetylcholine receptors ( $\mathrm{nAChRs),} \mathrm{which} \mathrm{are}$ the target of Lynx1 binding, tend to localize on cortical neurons and presynaptic thalamocortical terminals (Ibañez-Tallon et al., 2002; Disney et al., 2007). Consistently, our c-Fos studies in Lynx1KO mice showed that the experience of losing elicited higher c-Fos activation in the dACC and the MD compared with WT animals, brain regions that were previously shown to respond to previous wins to update dominance status (Zhou et al., 2017). Other studies that manipulated the social context to induce hierarchy plasticity have also shown activity of the PFC during times of changing social rank, confirming the PFC is a key region involved in updating dominance rank in response to social experience (Williamson et al., 2019). Together, our study suggests that a plasticity brake, known to regulate sensory cortex plasticity, can gate experience-dependent plasticity in the PFC circuit known to regulate dominance status. It should be noted that our study using c-Fos staining has limited temporal and spatial resolution. Future studies are necessary to examine to what extent c-Fos activation following forced lose accompanies changes in synaptic efficacy as well as network level oscillations between dACC and MD by incorporating electrophysiological methodology.

Our study also applied a pharmacological approach (VPA administration) to support a converging role of critical period plasticity regulators in inducing plasticity in social dominance hierarchy. While both genetic (Lynx1KO) and pharmacological (VPA) manipulations induced higher instability of mouse hierarchies, there were distinct differences between the outcomes of the two manipulations: Unlike Lynx1 loss, pharmacologically mediated reopening of the critical period through VPA treatment destabilizes baseline hierarchy instability (Figure 3). This difference could be due to the developmental timing of heightened cortical plasticity between these two models: Since Lynx1KO animals lack a break which normally facilitates the closure of the critical period, these animals show "openended" cortical plasticity while VPA treated adult WT animals would show a normal critical period closure, followed by an adult reopening of critical period plasticity. This developmental difference could lead Lynx1KO animals to compensate for elevated cortical plasticity across development through, for example, other known plasticity brakes such as PirB and NgR (McGee et al., 2005; Syken et al., 2006; Bavelier et al., 2010). Future studies are warranted to examine the contribution of these plasticity brakes to social dominance hierarchy plasticity. Adult increases in cortical plasticity following VPA treatment may sensitize animals to social experiences in the cage, leading to frequent updating of dominance relationships and high baseline hierarchy instability. Over time VPA-treated animals may show a stabilization of the baseline hierarchy and a similar pattern to Lynx1KO animals in which baseline hierarchies are stable and a manipulation of social experience is needed to unmask increased plasticity. Alternatively, VPA treatment may be a more potent destabilizer of hierarchy plasticity due to its mechanism of action and not the developmental timing of the manipulation. VPA has multiple mechanisms of action including enhancement of inhibition in the cortex and inhibition of histone deacetylases which can broadly alter the epigenome and lead to increased transcriptional plasticity (Phiel et al., 2001; Machado-Vieira et al., 2011). Whether through direct action on GABAergic signaling or through transcriptional responses due to increased acetylation, VPA is known to regulate oscillation activity in frontal cortex (Béla et al., 2007). Future studies are needed to assess whether GABAergic facilitation or HDAC inhibition or both simultaneously are required to disrupt hierarchy stability in adult mice. VPA is an anti-epileptic drug as well as an approved mood stabilizer and has been used as a treatment for Bipolar disorder (Phiel et al., 2001; Chiu et al., 2013). Future studies are warranted to determine if VPA treatment would provide an effective treatment to treat social deficits. It is also important to investigate the contribution of other pharmacological interventions known to reactivate sensory cortical plasticity in adulthood (e.g., selective serotonergic reuptake inhibitors, chondroitinase) (Pizzorusso et al., 2002; Maya Vetencourt et al., 2008) to social dominance hierarchy plasticity.

One of the limitations of this study is that we did not test if social experience impacts the opening or closure of sensitive period for social dominance hierarchy plasticity. Previous studies on critical period for visual cortex plasticity showed that dark rearing of mice from birth leads to prolonged visual critical period plasticity beyond juvenile period (Hensch, 2004). It would be interesting for future studies to examine to what extent pre-weaning (e.g., maternal deprivation) or post-weaning social experience (e.g., juvenile social isolation) impacts the timecourse of the sensitive period for social dominance hierarchy plasticity. Finally, our results demonstrate hierarchy stability modulation through plasticity regulators in male mice, however, female mice also form dominance hierarchies. Previous studies have shown female hierarchies are less dependent on experience (van den Berg et al., 2015), however, few studies have examined sex differences in neural mechanisms of dominance, social status, or the relative stability vs. plasticity of female hierarchies. 
Overall, our results demonstrate that PFC-dependent social behaviors such as mouse social dominance hierarchies are regulated by mechanisms that modulate critical period plasticity in primary sensory cortical areas. These findings provide insight into mechanisms through which increased behavioral plasticity may be achieved to improve therapeutic recovery from psychiatric disorders that are associated with social deficits.

\section{DATA AVAILABILITY STATEMENT}

The raw data supporting the conclusions of this article will be made available by the authors, without undue reservation.

\section{ETHICS STATEMENT}

The animal study was reviewed and approved by the Icahn School of Medicine at Mount Sinai Ethical Committee for animal research.

\section{REFERENCES}

Bavelier, D., Levi, D. M., Li, R. W., Dan, Y., and Hensch, T. K. (2010). Removing brakes on adult brain plasticity: from molecular to behavioral interventions. J. Neurosci. 30, 14964-14971. doi: 10.1523/jneurosci.4812-10.2010

Béla, C., Mónika, B., Márton, T., and István, K. (2007). Valproate selectively reduces EEG activity in anterior parts of the cortex in patients with idiopathic generalized epilepsy: a low resolution electromagnetic tomography (LORETA) study. Epilepsy Res. 75, 186-191. doi: 10.1016/j.eplepsyres.2007. 06.009

Bicks, L. K., Koike, H., Akbarian, S., and Morishita, H. (2015). Prefrontal cortex and social cognition in mouse and man. Front. Psychol. 6:1805. doi: 10.3389/ fpsyg.2015.01805

Bicks, L. K., Yamamuro, K., Flanigan, M. E., Kim, J. M., Kato, D., Lucas, E. K., et al. (2020). Prefrontal parvalbumin interneurons require juvenile social experience to establish adult social behavior. Nat. Commun. 11:1003.

Bukhari, N., Burman, P. N., Hussein, A., Demars, M. P., Sadahiro, M., Brady, D. M., et al. (2015). Unmasking proteolytic activity for adult visual cortex plasticity by the removal of Lynx1. J. Neurosci. 35, 12693-12702. doi: 10.1523/jneurosci. 4315-14.2015

Chiu, C.-T., Wang, Z., Hunsberger, J. G., and Chuang, D.-M. (2013). Therapeutic potential of mood stabilizers lithium and valproic acid: beyond bipolar disorder. Pharmacol. Rev. 65, 105-142. doi: 10.1124/pr.111.005512

David, H. A. (1987). Ranking from unbalanced paired-comparison data. Biometrika 74, 432-436. doi: 10.1093/biomet/74.2.432

Disney, A. A., Aoki, C., and Hawken, M. J. (2007). Gain modulation by nicotine in macaque v1. Neuron 56, 701-713. doi: 10.1016/j.neuron.2007.09.034

Gammell, M. P., De Vries, H., Jennings, D. J., Carlin, C. M., and Hayden, T. J. (2003). David's score: a more appropriate dominance ranking method than Clutton-Brock et al.'s index. Anim. Behav. 66, 601-605. doi: 10.1006/anbe.2003. 2226

Gervain, J., Vines, B. W., Chen, L. M., Seo, R. J., Hensch, T. K., Werker, J. F., et al. (2013). Valproate reopens critical-period learning of absolute pitch. Front. Syst. Neurosci. 7:102-102. doi: 10.3389/fnsys.2013.00102

Gordon, J. A., and Stryker, M. P. (1996). Experience-dependent plasticity of binocular responses in the primary visual cortex of the mouse. J. Neurosci. 16, 3274-3286. doi: 10.1523/jneurosci.16-10-03274.1996

Hensch, T. K. (2004). Critical period regulation. Annu. Rev. Neurosci. 27, 549-579. doi: 10.1146/annurev.neuro.27.070203.144327

Ibañez-Tallon, I., Miwa, J. M., Wang, H. L., Adams, N. C., Crabtree, G. W., Sine, S. M., et al. (2002). Novel modulation of neuronal nicotinic acetylcholine

\section{AUTHOR CONTRIBUTIONS}

LB and HM designed and analyzed experiments and wrote the manuscript with inputs from all authors. LB, MP, and AT performed all behavior experiments. LB and AT performed immunohistochemistry. SA and HM supervised LB. All authors contributed to the article and approved the submitted version.

\section{FUNDING}

This work was supported by the T32MH966785 to LB, and NIH R01MH117790-01 and P50MH096890 to SA, and NIH R01MH118297, R01MH119523, and the Simons Foundation/ SFARI (610850) to HM.

\section{ACKNOWLEDGMENTS}

We thank Hiroyuki Koike and Michael Leventhal for their helpful discussion.

receptors by association with the endogenous prototoxin lynx1. Neuron 33, 893-903. doi: 10.1016/s0896-6273(02)00632-3

Kingsbury, L., Huang, S., Wang, J., Gu, K., Golshani, P., Wu, Y. E., et al. (2019). Correlated neural activity and encoding of behavior across brains of socially interacting animals. Cell 178, 429-446 e416.

Lindzey, G., Winston, H., and Manosevitz, M. (1961). Social dominance in inbred mouse strains. Nature 191, 474-476. doi: 10.1038/191474a0

Machado-Vieira, R., Ibrahim, L., and Zarate, C. A. Jr. (2011). Histone deacetylases and mood disorders: epigenetic programming in geneenvironment interactions. CNS Neurosci. Ther. 17, 699-704. doi: 10.1111/j.1755-5949.2010.00203.x

Makinodan, M., Rosen, K. M., Ito, S., and Corfas, G. (2012). A critical period for social experience-dependent oligodendrocyte maturation and myelination. Science 337, 1357-1360. doi: 10.1126/science.1220845

Maya Vetencourt, J. F., Sale, A., Viegi, A., Baroncelli, L., De Pasquale, R., O’leary, O. F., et al. (2008). The antidepressant fluoxetine restores plasticity in the adult visual cortex. Science 320, 385-388. doi: 10.1126/science.1150516

McGee, A. W., Yang, Y., Fischer, Q. S., Daw, N. W., and Strittmatter, S. M. (2005). Experience-driven plasticity of visual cortex limited by myelin and Nogo receptor. Science 309, 2222-2226. doi: 10.1126/science.1114362

Miwa, J. M., Ibanez-Tallon, I., Crabtree, G. W., Sanchez, R., Sali, A., Role, L. W., et al. (1999). lynx1, an endogenous toxin-like modulator of nicotinic acetylcholine receptors in the mammalian CNS. Neuron 23, 105-114. doi: 10.1016/s0896-6273(00)80757-6

Miwa, J. M., Stevens, T. R., King, S. L., Caldarone, B. J., Ibanez-Tallon, I., Xiao, C., et al. (2006). The prototoxin lynx1 acts on nicotinic acetylcholine receptors to balance neuronal activity and survival in vivo. Neuron 51, 587-600. doi: 10.1016/j.neuron.2006.07.025

Morishita, H. (2020). A prefrontal social circuit vulnerable to juvenile social isolation. Neuropsychopharmacology 46, 229-230. doi: 10.1038/s41386-02000821-6

Morishita, H., and Hensch, T. K. (2008). Critical period revisited: impact on vision. Curr. Opin. Neurobiol. 18, 101-107. doi: 10.1016/j.conb.2008.05.009

Morishita, H., Miwa, J. M., Heintz, N., and Hensch, T. K. (2010). Lynx1, a cholinergic brake, limits plasticity in adult visual cortex. Science 330, 1238-1240. doi: 10.1126/science.1195320

Nelson, A. C., Kapoor, V., Vaughn, E., Gnanasegaram, J. A., Rubinstein, N. D., Murthy, V. N., et al. (2019). Molecular and circuit architecture of social hierarchy. bioRxiv [preprint] bioRxiv:838664doi:

Phiel, C. J., Zhang, F., Huang, E. Y., Guenther, M. G., Lazar, M. A., and Klein, P. S. (2001). Histone deacetylase is a direct target of valproic acid, a potent 
anticonvulsant, mood stabilizer, and teratogen. J. Biol. Chem. 276, 36734-36741. doi: 10.1074/jbc.m101287200

Pizzorusso, T., Medini, P., Berardi, N., Chierzi, S., Fawcett, J. W., and Maffei, L. (2002). Reactivation of ocular dominance plasticity in the adult visual cortex. Science 298, 1248-1251. doi: 10.1126/science.1072699

Putignano, E., Lonetti, G., Cancedda, L., Ratto, G., Costa, M., Maffei, L., et al. (2007). Developmental downregulation of histone posttranslational modifications regulates visual cortical plasticity. Neuron 53, 747-759. doi: 10.1016/j.neuron.2007.02.007

Sajo, M., Ellis-Davies, G., and Morishita, H. (2016). Lynx1 limits dendritic spine turnover in the adult visual cortex. J. Neurosci. 36, 9472-9478. doi: 10.1523/ jneurosci.0580-16.2016

Silingardi, D., Scali, M., Belluomini, G., and Pizzorusso, T. (2010). Epigenetic treatments of adult rats promote recovery from visual acuity deficits induced by long-term monocular deprivation. Eur. J. Neurosci. 31, 2185-2192. doi: 10.1111/j.1460-9568.2010.07261.x

Syken, J., Grandpre, T., Kanold, P. O., and Shatz, C. J. (2006). PirB restricts oculardominance plasticity in visual cortex. Science 313, 1795-1800. doi: 10.1126/ science. 1128232

Takesian, A. E., Bogart, L. J., Lichtman, J. W., and Hensch, T. K. (2018). Inhibitory circuit gating of auditory critical-period plasticity. Nat. Neurosci. 21, 218-227. doi: 10.1038/s41593-017-0064-2

Timmer, M., and Sandi, C. (2010). A role for glucocorticoids in the long-term establishment of a social hierarchy. Psychoneuroendocrinology 35, 1543-1552. doi: 10.1016/j.psyneuen.2010.05.011

van den Berg, W. E., Lamballais, S., and Kushner, S. A. (2015). Sex-Specific mechanism of social hierarchy in mice. Neuropsychopharmacology 40, 13641372. doi: 10.1038/npp.2014.319

Wang, F., Kessels, H. W., and Hu, H. (2014). The mouse that roared: neural mechanisms of social hierarchy. Trends Neurosci. 37, 674-682. doi: 10.1016/ j.tins.2014.07.005

Wang, F., Zhu, J., Zhu, H., Zhang, Q., Lin, Z., and Hu, H. (2011). Bidirectional control of social hierarchy by synaptic efficacy in medial prefrontal cortex. Science 334, 693-697. doi: 10.1126/science.1209951

Williamson, C. M., Klein, I. S., Lee, W., and Curley, J. P. (2019). Immediate early gene activation throughout the brain is associated with dynamic changes in social context. Soc. Neurosci. 14, 253-265. doi: 10.1080/17470919.2018.14 79303

Williamson, C. M., Lee, W., and Curley, J. P. (2016). Temporal dynamics of social hierarchy formation and maintenance in male mice. Anim. Behav. 115, 259-272. doi: 10.1016/j.anbehav.2016. 03.004

Yamaguchi, Y., Lee, Y. A., Kato, A., and Goto, Y. (2017a). The roles of dopamine D1 receptor on the social hierarchy of rodents and nonhuman primates. Int. J. Neuropsychopharmacol. 20, 324-335.

Yamaguchi, Y., Lee, Y. A., Kato, A., Jas, E., and Goto, Y. (2017b). The roles of dopamine D2 receptor in the social hierarchy of rodents and primates. Sci. Rep. $7: 43348$.

Yamamuro, K., Bicks, L. K., Leventhal, M. B., Kato, D., Im, S., Flanigan, M. E., et al. (2020). A prefrontal-paraventricular thalamus circuit requires juvenile social experience to regulate adult sociability in mice. Nat. Neurosci. 23, 1240-1252. doi: 10.1038/s41593-020-0695-6

Yang, E. J., Lin, E. W., and Hensch, T. K. (2012). Critical period for acoustic preference in mice. Proc. Natl. Acad. Sci. U.S.A. 109(Suppl. 2), 17213-17220. doi: 10.1073/pnas.1200705109

Zhou, T., Sandi, C., and Hu, H. (2018). Advances in understanding neural mechanisms of social dominance. Curr. Opin. Neurobiol. 49, 99-107. doi: 10.1016/j.conb.2018.01.006

Zhou, T., Zhu, H., Fan, Z., Wang, F., Chen, Y., Liang, H., et al. (2017). History of winning remodels thalamo-PFC circuit to reinforce social dominance. Science 357, 162-168. doi: 10.1126/science.aak9726

Conflict of Interest: The authors declare that the research was conducted in the absence of any commercial or financial relationships that could be construed as a potential conflict of interest.

Copyright (C) 2021 Bicks, Peng, Taub, Akbarian and Morishita. This is an open-access article distributed under the terms of the Creative Commons Attribution License (CC BY). The use, distribution or reproduction in other forums is permitted, provided the original author(s) and the copyright owner(s) are credited and that the original publication in this journal is cited, in accordance with accepted academic practice. No use, distribution or reproduction is permitted which does not comply with these terms. 